C2008 IEEE. Personal use of this material is permitted. However, permission to reprint/republish this material for advertising or promotional purposes or for creating new collective works for resale or redistribution to servers or lists, or to reuse any copyrighted component of this work in other works must be obtained from the IEEE. 


\title{
Transport Service Ontology and Its Application in the Field of Semantic Search
}

\author{
Hai Dong, Farookh Khadeer Hussain, Elizabeth Chang \\ Digital Ecosystems and Business Intelligence Institute \\ Curtin University of Technology \\ Perth, Australia \\ E-mail: \{hai.dong, farookh.hussain, elizabeth.chang\}@cbs.curtin.edu.au
}

\begin{abstract}
Transport is a crucial component in logistics, which joints each activity in the logistics chain. The performance of logistics heavily relies on the efficiency of transport services. However, until now there has not been a clear definition about transport services. Moreover, along with the rapid rise of fuel prices, the input-output ratio of transport has been trapped in an unreasonable situation, which causes the poor performance of logistics. Therefore, the research on transport service and transport service efficiency is urgently required. In this paper, we propose to use semantic web technologies to construct a conceptual model of transport services and thus present a clear structure of the transport service hierarchy. Furthermore, we propose a semantic search engine based on this transport service ontology, in order to provide an efficient tool for users to query transport service providers. This design can also be seen as a potentially alternative approach for solving the issue of transport service inefficiency.
\end{abstract}

Keywords-transport service ontology; semantic search; semantic search engine

\section{INTRODUCTION}

Transport is a crucial component in logistics, which joints each activity in the logistics chain [10]. It is believed that more than one third of cost in logistics is spent on transport. In addition, the performance of logistics highly relies on the efficiency of transport services. However, until now there has not been a clear definition about transport services. Moreover, along with the rapid rise of fuel price, the input-output ratio of transport has been trapped in an unreasonable situation, which causes the poor performance of logistics. Therefore, the research on transport services and transport service efficiency is urgently required.

In this paper, we propose to use semantic web technologies to construct the conceptual model of transport services and thus present a clear structure of the transport service hierarchy. Furthermore, we propose a semantic search engine based on this transport service ontology, in order to provide an efficient tool for users to query transport service providers. This design can also be seen as a potentially alternative approach for solving the issue of transport service inefficiency.

The whole paper structure is organized as follows: first of all we review the literature regarding ontology and semantic search; then a notation system for our transport service ontology representation is presented; by means of the notation system, we deliver a transport service ontology concept hierarchy and a transport service ontology, followed by the conceptual model of the semantic search engine based on the ontology; then we reveal the implementation details about the transport service semantic search engine; finally we discuss the limitations about this project and plan our future work.

\section{LITERATURE REVIEW: ONTOLOGY AND SEMANTIC SEARCH}

In this section, we will make a brief review on literatures with respect to ontology and semantic search.

\section{A. Ontology}

Ontology is defined as "an explicit machine-readable specification of a shared conceptualization" [5]. Ontology provides common vocabularies for both humans and computers, which supports semantics for knowledge sharing between them [4]. The application of ontology spreads into the field of semantic web, health science, e-commerce and so on, in order to conceptualize and standardize specific domain knowledge, and to solve interoperability problems emerged in the knowledge sharing among cross-domains.

\section{B. Semantic Search}

Guha et al. categorize the existing search engines into two categories - navigational search and research search. In the first category, users enter keywords to query documents where the keywords coexist, which cannot be used to denote concepts. In the second category, users use words to denote certain concepts, which could help search engines to understand users' query intentions. Semantic search concentrates on the second form of search [8].

Currently, many semantic search engines are designed and implemented in order to adapt to different working environments, and the mechanisms that realize these search engines are thus distinct. Guha and $\mathrm{McCool}$ deliver a semantic search engine in TAP system - a comprehensive semantic web system [7]. The query language for semantic search in TAP is called GetData interface, which allows programs to visit 
properties of a resource in a semantic graph. Liu, Shen and Liao invent an e-service platform integrated with semantic search for e-service metadata [9]. E-service metadata refers to descriptions about e-services and providers, which is to publish and to discover e-services. There are two types of metadata in the system: business level metadata - the description of eservice providers; service level metadata - the description of basic information about e-service. The authors adopt Universal Description, Discovery and Integration which is a web service standard to register and search e-services. Dichev and Dicheva propose a view-based semantic search engine in the context of topic-centered learning repository, by means of the extension of the Topic Maps (TM) model - which is a lightweight ontology model constructed by topics and relationships between topics [3]. Wang et al. project a semantic search methodology to retrieve information from normal tables, which has three main steps: identifying semantic relationships between table cells; converting tables into data in the form of database; retrieving objective data by query languages [11].

\section{NOTATION SYSTEM FOR TRANSPORT SERVICE ONTOLOGY REPRESENTATION}

The notation system utilized in the ontological representation is based on the work of Chang et al., which consists of three basic notations as Table 1 [2]. Although most researchers use UML to represent ontology models, due to its complex symbol system, UML cannot efficiently help people without software engineering knowledge to better understand the shared knowledge. This notation system simplifies the symbols and its symbols are closer to the principle of ontology, which is the combination of shared concepts and relationships between concepts [6].

\section{TRANSPORT SERVICE ONTOLOGY}

In this section, the hierarchy of transport service ontology concepts and the transport service ontology are represented.

\section{A. Hierarchy of Transport Service Ontology Concepts}

The structure of transport service ontology concepts is a four-layer hierarchy (Fig. 1). The first layer is the root of the hierarchy, which represents the abstract concept of all services in the transport domain. The second layer is the preliminary specialization for the abstract transport service concept, which classifies four categories of transport service concept - air transport, rail transport, road transport, and shipping services.
TABLE I. ONTOLOGICAL Notation SYSTEM

\begin{tabular}{|c|l|}
\hline \multicolumn{1}{|c|}{$\begin{array}{c}\text { Ontology } \\
\text { Notations }\end{array}$} & \multicolumn{1}{|c|}{ Semantics of the Notations } \\
\hline & $\begin{array}{l}\text { Double-field Box represents the Ontological } \\
\text { Concepts. }\end{array}$ \\
\hline- relation $--^{-}$ & $\begin{array}{l}\text { A dotted line represents Ontology Concept } \\
\text { Association Relation which represents a Concept } \\
\text { is closely related to another concept. The } \\
\text { relationship name can be noted above the dotted } \\
\text { line. }\end{array}$ \\
\hline$\rightarrow$ & $\begin{array}{l}\text { Solid-arrow line represents Generalization and } \\
\text { Specification relation, which is a relation between } \\
\text { Upper Generic Concept and Lower Specific } \\
\text { Concept. }\end{array}$ \\
\hline
\end{tabular}

These can be also regarded as the four basic sub-domains of transport services. The service concepts in this layer are still considered as abstract. The third layer is the further specialization for the abstract transport service concepts in the second layer, which represents the services in each basic subdomain of transport services. In this layer, the service concepts could be regarded as concrete or abstract concepts. For each concrete service concept, it corresponds to the actual services in the real social environment; for each abstract service concept, it has further specializations which are all concrete services in the fourth layer. In conclusion, the service concepts in the first and second layer are all abstract concepts, which execute the function of service domains definition, the service concepts in the third layer are hybrid concepts between concrete concepts and abstract concepts, which are used to restrict service domain and correspond to the actual services in the real world; the service concepts are concrete concepts in the bottom layer.

\section{B. Transport Service Ontology}

The Transport Service Ontology is defined as the conceptualization of the Transport Service, which is identified by a Transport Service Name Concept, defined by a Transport Service Description, restricted by a Transport Service Area, and finally provided by a Transport Service Provider who is located by a Transport Service Provider Address and a Transport Service Provider Contact Detail (Fig. 2).

We present the Transport Service Ontology as the combination of the ontology name and a tuple where the elements of the tuple can be complex elements as defined

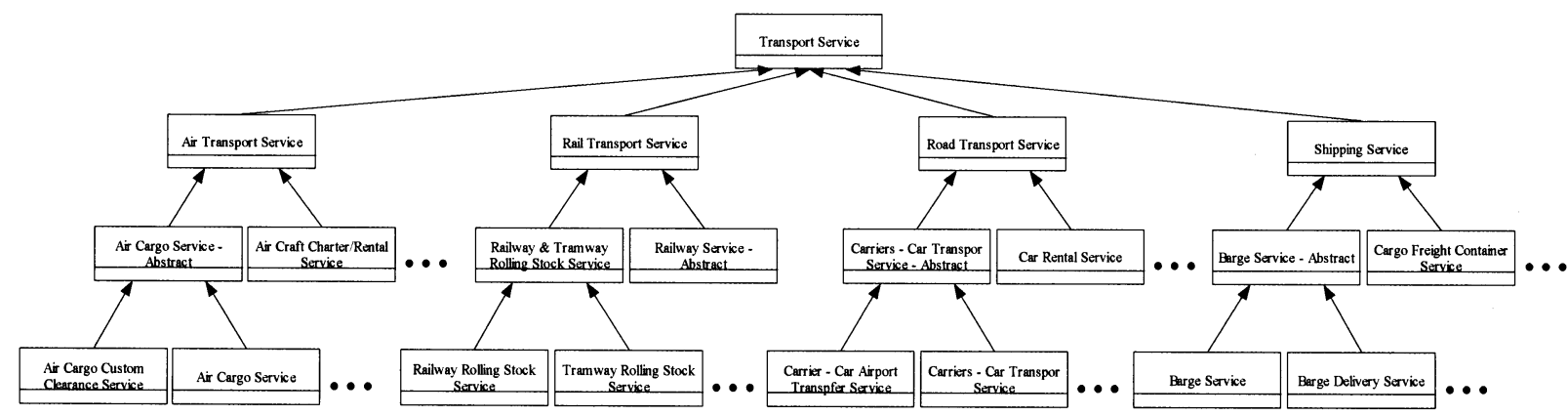

Figure 1. Transport service ontology concept hierarchy 


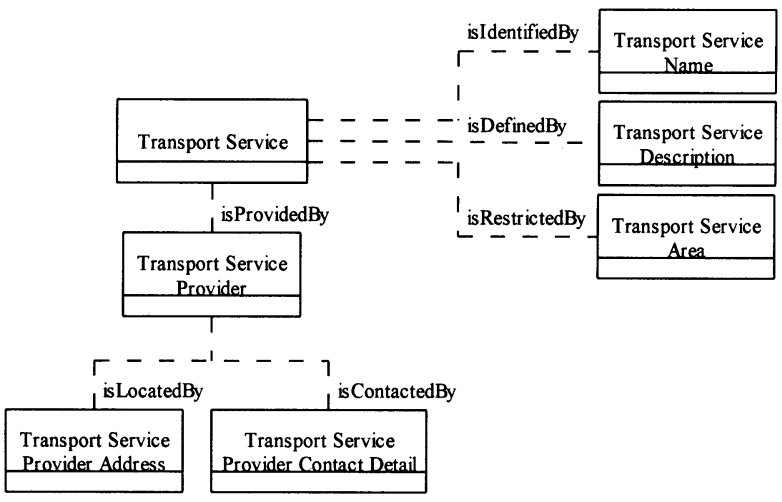

Figure 2. Transport service ontology

below:

Transport Service [Transport Service Name, Transport Service Description, Transport Service Area, Transport Service Provider, Transport Service Provider Address, Transport Service Provider Contact Detail] where

Transport Service Name refers to the name that can be used to uniquely identify a service.

Transport Service Description refers to the detailed text description with regards to the content of a service.

Transport Service Area refers to the scope of area where a transport service can be implemented.

Transport Service Provider refers to the name of the person or organization that provides a service.

Transport Service Provider Address refers to the address where a service provider can be located.

Transport Service Provider Contact Detail refers to the information regarding how a service provider is contacted, for instance, mail box, phone number, fax number, website and so on.

The transport service ontology is the definition of a transport service concept in the root of the transport service concept hierarchy. As children concepts, all other concepts in this hierarchy inherit the definition according to their specific domain knowledge, which means that domain-specific contents are added into their individual definitions.

\section{ApPlication of Transport SERVice Ontology in THE FIELD OF SEMANTIC SEARCH}

In this section, we propose a semantic search engine adopting the transport service ontology. This implementation will include two main parts, namely constructing a transport service knowledgebase and a transport service search engine.

\section{A. Transport Service Knowledgebase}

The transport service knowledgebase contains two primary components - the transport service ontology and transport service metadata. The former is already described in the prior section. For the latter, we design a standard metadata format -

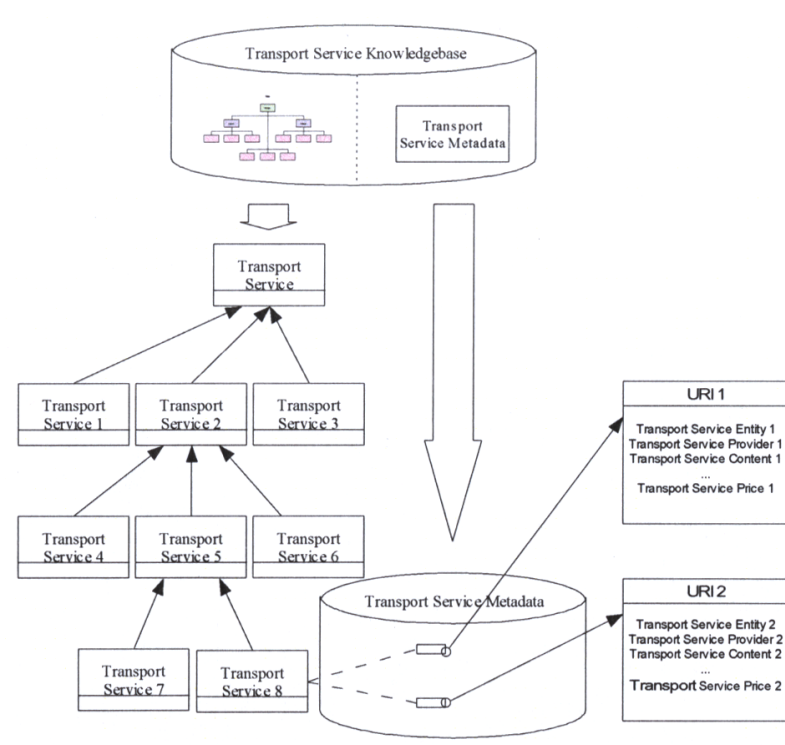

Figure 3. System architecture of transport service knowledgebase

Transport Service Entity (TSE) format. For the association between the transport service ontology and transport service metadata, an algorithm is designed in order to semantically match them. The algorithm will calculate the semantic similarity values between the concrete transport service concepts in the hierarchy and the transport service metadata. Meanwhile, a threshold is configured for the semantic matching. Once a similarity value is beyond the threshold, the corresponding transport service concept and metadata can be regarded as semantically related. The uniform resource identifier (URI) of the metadata will be passed to the concept, which can be seen as the accomplishment of the association process. The system architecture of transport service knowledgebase can be seen in Fig. 3.

\section{B. A Semantic Search Engine Based on the Transport Service Knowledgebase}

Based on the transport service knowledgebase, we design a semantic search algorithm originated from the Case-Based Reasoning (CBR) algorithm [1]. Similar to the algorithm used for associating between transport service concepts and metadata in the transport service knowledgebase, the semantic search algorithm can compute the semantic similarity values between a query and all ontology concepts, but the difference is that the computing involves not only concrete concepts but also abstract concepts. Also, a threshold is set up before the search, and all concepts whose semantic similarity values are beyond the value are selected and ranked according to their semantic similarity values. Once an abstract concept is retrieved, it will show all its subconcepts, which can help users to further denote their query intentions; once a concrete concept is retrieved, all metadata that semantically link to the concept will be presented to users. 


\section{IMPLEMENTATION}

Similar to the conceptual model design phase, the prototype implementation phase contains two sub-processes, which are transport service knowledgebase construction and semantic search engine implementation.

The first process is realized by using Protégé-owl, which can be seen in Fig. 4. In addition, the domain knowledge used for constructing the transport service concept hierarchy is obtained from the Australian Yellow Pages ${ }^{\circledR}$ website and its linked transport companies. We also use this website as the primary data source to obtain transport service metadata. From the services under the transport service category in this website, all transport service information are extracted and stored in the form of TSE format, and then the semantic matching algorithm is executed to match transport service concepts with metadata. Finally, for each concept, the URIs of all matched metadata are then stored in the knowledgebase.

To realize the second implementation process, we use java as the primary tool to design the GUI of semantic search engine. The screenshot of the GUI can be found in Fig. 5. The whole search workflow is follows:

- Before users start a search, there are two query options for users to choose, which are "search for keywords" and "browse by category".

- If users choose the former option and enter query words, as seen in Fig. 5, the bottom left GUI frame will display all the matched concepts ranked by their semantic similarity values in percentage. If the displayed concept is a concrete concept, the number in the round bracket behind its concept name can indicate how many service metadata semantically associate to the concept; if it is an abstract concept, all its subconcepts are unfolded and ranked according to their semantic similarity values, once the concept is clicked.

- The second query option can display the whole hierarchy of transport service concepts. Thus users can choose the path by themselves to denote their querying concepts.

- Once a concrete transport service concept is clicked, the

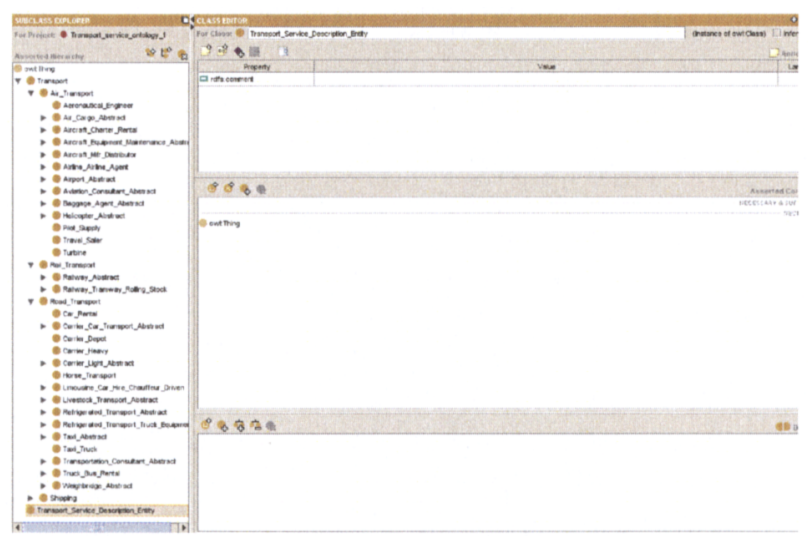

Figure 5. Screenshot of transport service ontology in Protégé-owl

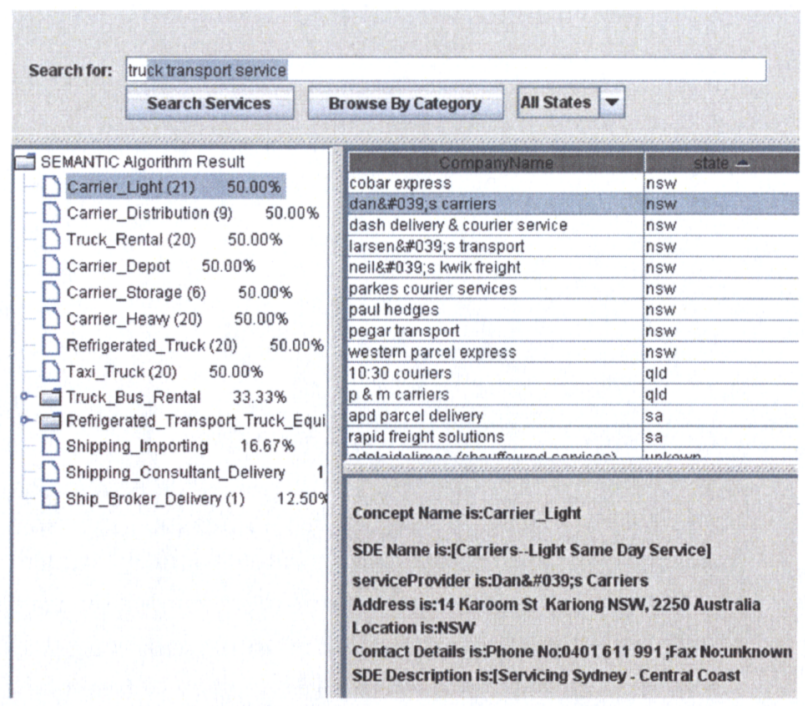

Figure 4. Screenshot of semantic search engine GUI

name of all its semantically related service providers are displayed and listed in the middle right GUI frame, as well as ranked in alphabetic order or state name order.

- Finally, if a user clicks a service provider name in the list, the information regarding the transport service metadata will be displayed in the bottom right GUI frame.

\section{CONCLUSION}

In this paper, we propose a transport service ontology, in order to define the conceptual model of transport services. To help users solve the issue of transport service inefficiency, we design a semantic search engine by adopting the ontology as the backbone of a transport service knowledgebase, which could help users rapidly query transport service providers.

The limitations of this project can be concluded as below:

- The data source of transport service metadata is only from the Australian Yellow Pages ${ }^{\circledR}$, which has limited query scope.

- The semantic search engine is only in experimental phase, which has not been implemented on the internet.

Against the two limitations, in the future work, our research will concentrate on seeking more transport service data source from other websites to enrich the content of our service knowledgebase, and publishing the transport service search engine on the internet for testing purpose.

\section{ACKNOWLEDGMENT}

I would like to express our gratitude to Professor Chang and Dr. Hussain for their distinguished book. I also appreciate the assistance of all DEBII members, especially Wei Liu who is the programmer responsible for the whole prototype implementation.

\section{REFERENCES}


[1] D. C. J. Carthy, A. Drummond, J. Dunnion, and J. Sheppard, "The use of data mining in the design and implementation of an incident report retrieval system," in Systems and Information Engineering Design Symposium, Charlottesville, 2003, pp. 13-18.

[2] E. Chang, T. Dillon, and F. Hussain, Trust and Reputation for Service Oriented Environments-Technologies for Building Business Intelligence and Consumer Confidence: John Wiley \& Sons, 2005.

[3] C. Dichev and D. Dicheva, "View-Based Semantic Search and Browsing," in WI '06, 2006.

[4] D. Fensel, F. v. Harmelen, M. Klein, and H. Akkermans, "On-ToKnowledge: Ontology-based tools for knowledge management," in The eBusiness and eWork 2000 Conference (EMMSEC 2000), P. T. Kidd, Ed. Madrid: Cheshire Henbury, 2000.

[5] A. Gómez-Pérez and O. Corcho, "Ontology specification languages for the semantic web," IEEE Intelligent Systems, vol. 17, pp. 54-60, 2002.
[6] N. Guarino, "Ontology-Driven Conceptual Modelling," in The 21st International Conference on Conceptual Modeling - ER 2002, Tampere, Finland, 2002

[7] R. Guha and R. McCool, "TAP: a Semantic Web platform," Computer Networks, vol. 42, pp. 557-577, 2003.

[8] R. Guha, R. McCool, and E. Miller, "Semantic search." vol. 2008: W3C, 2003

[9] D.-R. Liu, M. Shen, and C.-T. Liao, "Designing a composite e-service platform with recommendation functions," Computer Standards \& Interfaces, vol. 25, pp. 103-117, 2003.

[10] Y.-y. Tseng, W. L. Yue, and M. A. P. Taylor, "The role Of transportation in logistics chain," in the 2005 Eastern Asia Society for Transportation Studies, 2005, pp. 1657-1672.

[11] H. L. Wang, S. H. Wu, I. C. Wang, C. L. Sung, W. L. Hsu, and W. K Shih, "Semantic search on Internet tabular information extraction for answering queries," in $C I K M^{\prime} 00$, McLean, 2000, pp. 243-249. 